\title{
Osteítis actinomicótica del pulgar: diagnóstico y tratamiento
}

\author{
B. Gago Vidal ${ }^{(1)}$, A. Álvarez Jorge ${ }^{(2)}$, F. Martelo Villar ${ }^{(3)}$ \\ Servicio de Cirugía Plástica y Reconstructiva \\ Complexo Hospitalario Universitario A Coruña \\ (1) MÉdico RESIDENTE \\ (2) MÉDICO ADJUNTO \\ (3) JEFE DE SERVICIO
}

Correspondencia:

Dr. Bruno Gago Vidal

Servicio de Cirugía Plástica y Reconstructiva

Complexo Hospitalario Universitario A Coruña

C/ Xubias de Arriba, 84

15006 A Coruña

Teléfono (+34) 981178000

e-mail: brunogagoplastica@gmail.com

Se presenta el caso de un paciente con actinomicosis ósea y destrucción total de la falange distal del pulgar derecho. El tratamiento consistió en antibioterapia y desbridamiento en un primer tiempo con posterior reconstrucción del pulgar mediante colgajo compuesto pediculado radial del antebrazo.

Palabras clave: actinomicosis, pulgar, reconstrucción.
A patient with osseus actinomycosis and total bone destruction of the distal phalanx of the right thumb is presented. The treatment was antibiotherapy and debridment and posterior reconstruction procedure with a composite distally pedicled radial forearm flap.

Key words: actinomycosis, thumb, reconstruction.

\section{CASO CLÍNICO}

e presenta el caso de un paciente varón de 67 años, ganadero de profesión. Un año antes de acudir a nuestro servicio presentó herida (laceración) en primer dedo de mano derecha tras mordedura humana. En las siguientes 24 horas notó hinchazón y dolor local. Tras tratamiento antibiótico vía oral con tetraciclinas durante una semana el paciente refirió mejoría clínica con persistencia de eritema.

Siete meses después, la porción distal del pulgar aparecía aumentada de tamaño y consisten- cia blanda. Tras varios días de tratamiento con el mismo antibiótico el paciente refirió ligera mejoría.

Acude al hospital remitido por su médico de atención primaria por presentar en el pulgar derecho zona de tumefacción fluctuante con induración y eritema con mala respuesta a antibioterapia (Figura 1). El estudio radiológico de la mano reveló la existencia de severa osteomielitis y destrucción completa de la falange distal del pulgar derecho (Figura 2).

Se procedió a drenar el área de fluctuación del pulgar y las zonas más afectadas, incluyen- 


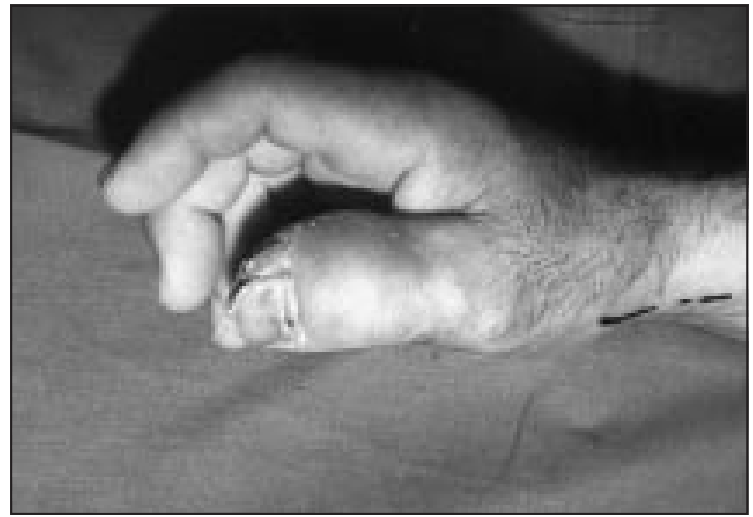

Figura 1: Aspecto prequirúrgico del pulgar derecho.

do hueso, fueron desbridadas. El pus era espeso, amarillo-marrón y contenía varios gránulos blanquecinos-amarillentos. Se hizo tinción Gram del mismo que mostró estructuras filamentosas Gram positivas y cocos Gram positivos en cadenas. La preparación de hidróxido de potasio al $10 \%$ fue positiva por gránulos de sulfuro. El cultivo del pus mostró la presencia de Actinomyces israelii y alpha-Streptococcus.

El paciente fue tratado con dosis altas de Penicilina $G$ intravenosa. A las dos semanas la herida estaba limpia pero la falange distal estaba totalmente reabsorbida y la proximal expuesta. Se realiza reconstrucción quirúrgica del pulgar con un colgajo neurocutáneo del antebrazo basado en la arteria radial y antibioterapia con penicilina vía oral durante un período de 10 meses tras el alta hospitalaria.

Un año más tarde el paciente presentaba un pulgar reconstruído con buena función y sin signos de infección (Figura 3).

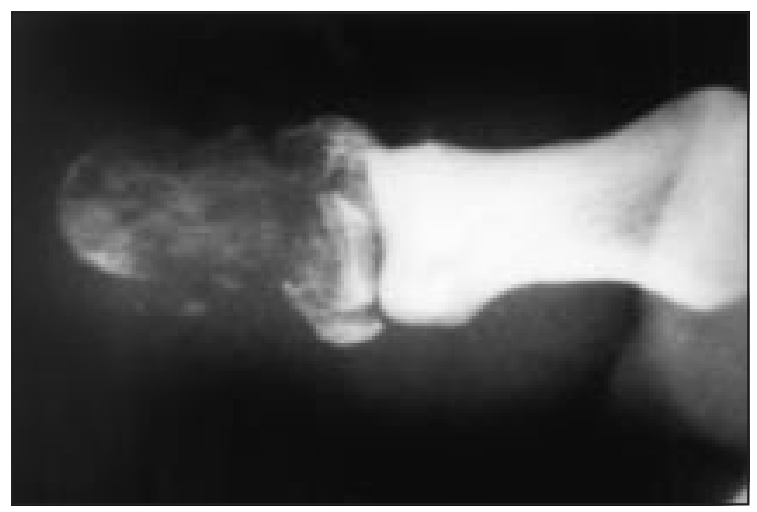

Figura 2: Radiografía que muestra la destrucción ósea de la falange distal.

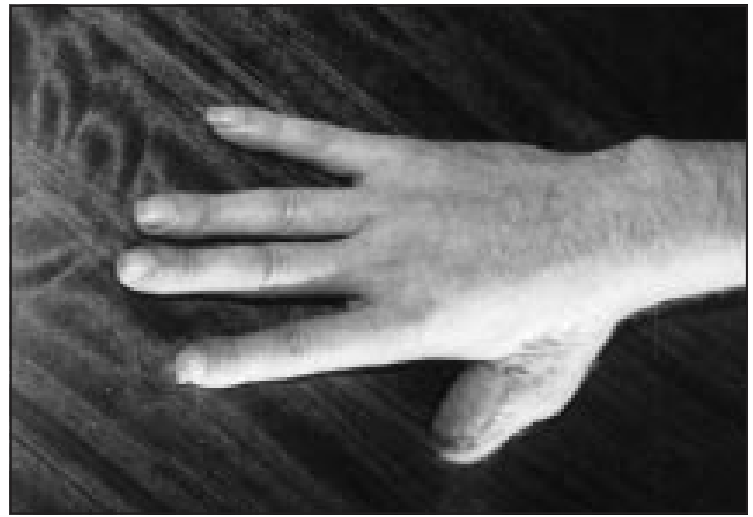

Figura 3: Resultado al año tras procedimiento reconstructivo.

\section{DISCUSIÓN}

En los últimos años pocos han sido los casos de actinomicosis descritos en humanos ${ }^{1,2,3}$. En la mayoría de los casos publicados, la infección por Actinomyces en la mano se desarrolló tras un puñetazo, al golpearse el paciente con la dentadura del adversario. Esto provoca una herida lacerativa contaminada por Actinomyces israelii, habitualmente presente en caries. El tejido blando infectado afecta posteriormente al hueso por contigüidad ${ }^{4}$.

En todos los casos descritos en la literatura la terapia consistió en desbridamiento quirúrgico combinado con terapia antibiótica prolonga$\mathrm{da}^{5}$. La penicilina se considera el antibiótico de elección para el tratamiento de la actinomicosis. Debe ser administrada por vía parenteral en altas dosis inicialmente continuándose con antibioterapia vía oral por un período de 6 a 12 meses $^{6}$.

Con la falange completamente destruida y con el proceso infeccioso resuelto, la indicación era la reconstrucción del pulgar. Debido a la patología vascular crónica que el paciente presentaba en miembros inferiores declinamos optar por una transferencia de pulgar. El tratamiento reconstructivo utilizado fue el colgajo compuesto de la arteria radial pediculado distalmente incluyendo una rama cutánea del radial que se anastomosó con el nervio colateral cubital del pulgar?

No se incluyó hueso en el colgajo para el alargamiento del pulgar pues la longitud obtenida tras la reconstrucción se consideró suficiente. De este modo nuestro paciente consiguió realizar una pinza completa y evitamos complicaciones postoperatorias como fractura del radio. 


\section{BIBLIOGRAFÍA}

1. Rushforth GF, Eykyn SJ. Actinomycosis of the hand. Hand, 1982; 14: 194-7.

2. Blinkhorn RJ, Strimbu V, Effron D, Spagnuolo PJ. «Punch» Actinomycosis causing osteomielytis of the hand. Arch Intern Med, 1988; 148: 2668-70.

3. Mert A, Bilir M, Bahar H, Torun M, Tabak F, Ozturk R, et al. Primary actinomycosis of the hand: a case report and lite- rature review. Int $\mathrm{J}$ Infect Dis. 2001; 5: 112-4

4. Winner HI, Punch actinomycosis. Lancet, 1960; 2: 907.

5. Kocher MS, Cooms CJ, Upton $\mathrm{J}$ III. Actinomycetoma of the phalanx in an immunocompromised patient. J Hand Surg Am, 1996; 21: 515-7.

6. Lerner PI. Nocardia species: Actinomyces and Arachnia species. En Mandell GL, Do- uglas RG Jr, Bennet JE (Eds). Principles and Practice of infectious Diseases, 2 ed. New York: John Wiley \& Sons Inc, 1985: 1423-33.

7. Foucher G, Genechten F, Merle $\mathrm{N}$, Michon J. A compound radial artery forearm flap in hand surgery: an original modification of the chinese forearm flap. Br J Plast Surg, 1984; 37: 139-48. 